\title{
Accidentalidad Laboral en el Sector de la Construcción: el Caso del Distrito de Cartagena de Indias (Colombia), Periodo 2014-2016
}

\author{
Elías A. Bedoya ${ }^{(1)^{\star}}$, Carlos A. Severiche ${ }^{(2)}$, Dario D. Sierra ${ }^{(2)}$ e Irma C. Osorio ${ }^{(2)}$ \\ (1) Fundación Universitaria Tecnológico Comfenalco, Coordinador de investigación del Programa de \\ Tecnología en Seguridad e Higiene Ocupacional, Grupo de Investigación CIPTEC, Cartagena de Indias, \\ Bolívar-Colombia (e-mail: ebedoya@tecnologicocomfenalco.edu.co) \\ (2) Fundación Universitaria Tecnológico Comfenalco, Facultad de Ingeniería, Programa de Tecnología en \\ Seguridad e Higiene Ocupacional, Cartagena de Indias, Bolívar-Colombia
}

* Autor a quien debe ser dirigida la correspondencia

Recibido May. 24, 2017; Aceptado Ago. 3, 2017; Versión final Sep. 15, 2017, Publicado Feb. 2018

\begin{abstract}
Resumen
Se realizó un análisis de accidentalidad laboral identificando los riesgos asociados al proceso de pilotaje, excavaciones y fundición de placas utilizado en 118 trabajadores accidentado, sin fatalidades, de seis empresas del sector de la construcción de la ciudad de Cartagena de indias, en Colombia. La investigación es de tipo descriptiva, ya que especifica las actividades de personas, grupos, y entorno respecto al fenómeno de accidente de trabajo. Además, utiliza la observación y análisis documental de investigaciones anteriores como método para reunir información sobre lo que acontecía en las empresas constructoras. Los golpes (golpeado por y golpeado contra) tuvieron la mayor prevalencia con frecuencias del $50 \%, 45 \%$ y $52 \%$ en los respectivos años en que se desarrolló el estudio (2014-2016). El año 2014 reportó mayor cantidad de accidentes con fractura (14\%), siendo estos los más incapacitantes del grupo de eventos de accidente analizados.
\end{abstract}

\section{Work Accidents in the Construction Sector: the Case of the District of Cartagena de Indias (Colombia), Period 2014-2016}

\begin{abstract}
An analysis of work accident was carried out to identify the risks associated with the process of piloting, excavation and casting used in 118 injured workers, without fatalities in six companies of the construction sector in the city of Cartagena de Indias, Colombia. The research is descriptive, as it specifies the activities of people, groups, and environment regarding the phenomenon of work accident. In addition, it uses the observation and documentary analysis of previous research, as the method to gather information on what was occurring in the construction companies. Blows (hit by and hit against) had the highest prevalence with frequencies of $50 \%, 45 \%$ and $52 \%$ in the respective years in which the study was developed (2014-2016). The year 2014 reported a greater number of accidents with fracture (14\%), being these the most incapacitating in the group of accident events analyzed.
\end{abstract}

Keywords: accident, construction, physical disability, injury 


\section{INTRODUCCIÓN}

En las condiciones de trabajo se sintetiza la forma como la actividad laboral determina la vida humana, en ellas se debe tener en cuenta los peligros a los cuales está sometido el trabajador, debido a que la construcción es una de las mayores industrias del mundo, agrupa actividades como ingeniería civil, demolición, renovación, reparación y mantenimiento dejando expuestos trabajadores a una gran variedad de situaciones peligrosas, causantes de accidentes como los ocurridos en distintos entornos de la construcción, tales como caídas $(54,1 \%)$, golpeadas por objetos lanzados o caídos $(12,9 \%)$, colapsos estructurales $(9,9 \%)$ y electrocuciones $(7,5 \%)$ siendo las $15: 00$ y $17: 00$ horas $(22.6 \%)$, las $10: 00-12: 00$ horas $(18.7 \%)$ y justo después de la hora del almuerzo (9.9\%) las prolíficas en la ocurrencia de accidentes en el contexto turco donde además las caídas representan el mayor número de accidentes $(54,7 \%)$, con impactos por proyección, proyección o caída de objetos (13,2\%), colapso constructivo / estructural (10,5\%) y exposición a electricidad (6,4\%) como segunda, tercera y cuarta Causas más frecuentes de accidentes (Gómez et al., 2017). Donde además los trabajadores no calificados, las tres ocupaciones con mayor número de lesiones mortales fueron: pintores y yeseros $(9,6 \%)$, carpinteros y andamios de madera (6,6\%) y otros artesanos como soldadores $(6,6 \%)$. Las muertes de terceros y niños también fueron consideradas comunes en la construcción (Bedoya et al., 2017). Las caídas a través de las superficies del techo se asociaron con una falta de andamios de cumplimiento. Las caídas reportadas se asocian con sobreesfuerzos y control inusual en el uso de escaleras además de herramientas inseguras (Gürcanli y Müngen, 2013; Vega et al., 2017).

La industria de la construcción en la India ha logrado un crecimiento extenso en todo el mundo en particular en las últimas décadas. Para que un proyecto de construcción tenga éxito, la seguridad de las estructuras, así como la del personal son importantes. Esto para evitar la generación de eventos como la muerte de trabajadores que según cifras publicadas estuvieron alrededor del 1,60\%, con un promedio de 16.03 en pequeñas obras, pérdida de miembros $2 \%$, fracturas $10,47 \%$, lesiones en general $44,10 \%$, infecciones de la piel $8.08 \%$, falencias visuales y auditivas $2.07 \%$ y en general la media de accidentes de 10,07 con edades promedio de los trabajadores afectados de 32 años, tales afectaciones fueron causados por causados por factores como colapso de partes de edificios y masas de tierra, caída de objetos y piezas de trabajo en trabajadores, caída de personas desde alturas, escaleras y mecanismos de carga, descarga y transporte de elementos de construcción, trabajo en máquinas e incluso voladura con explosivos (Kanchana et al., 2015). Los accidentes de trabajo en la industria de la construcción China son frecuentes y pueden producir discapacidades permanentes y muertes acompañados por lesiones más frecuentes como fracturas, trauma, contusiones, contusiones, moretones y abrasiones, provocados por las condiciones físicas de andamios y escaleras utilizados para realizar tareas de construcción en altura. También es referida la peligrosidad que revisten los andamios y escaleras pueden ser muy peligrosas cuando se usan incorrectamente (Chan et al., 2008). Investigaciones en zonas del sur de Australia, donde el factor meteorológico se ha relacionado con la accidentalidad, muestra que los trabajadores varones han sufrido una proporción desproporcionadamente mayor de accidentes graves en comparación con las trabajadoras durante épocas de verano, debido a subestimar el riesgo y sobreestimar su capacidad para hacer frente a ella, lo cual ha incrementado el gasto medio de los trabajadores masculinos por accidentes de este tipo (Rameezdeen, et al., 2017).

Los servicios de salud y seguridad en el trabajo del contratista general y la falta de una mayor participación de los contratistas generales en las cuestiones de salud y seguridad de los subcontratistas, y el control del cumplimiento por parte de los subcontratistas de los requisitos de salud y seguridad en el trabajo, son otra problemática de los prestadores de servicios de construcción en europa, al no proveer las mejores condiciones a sus trabajadores los exponen a más riesgos de accidentalidad (Dąbrowski, 2015).

Los factores más comunes asociados con la accidentalidad en el sector de la construcción y otras similares fueron abordados por investigadores australianos que consideraron que las características individuales, las condiciones locativas y las condiciones meteorológicas presentes, además el $25 \%$ de los estudios indican que los andamios y escaleras utilizados para tareas de construcción son una manera riesgosa que podría causar muertes de trabajadores de este sector (Nadhim et al., 2016). Eventos como tropiezos, caídas al mismo nivel, distinto nivel y caída de altura se consolidan como los incidentes en la construcción más significativos por su ya reconocida severidad en el daño causado en los trabajadores incluida la fatalidad en el contexto internacional (Work-Related Injuries and Fatalities Involving A Fall from Height, Australia, 2013; Bedoya, 2015).

En la actualidad la accidentalidad genera consecuencias negativas en la integridad de los trabajadores y en la empresa. Por lo tanto, la seguridad y salud en el trabajo como alternativa de solución, pretende mejorar y mantener la calidad de vida y salud de los trabajadores, procurando aumentar la productividad y eficacia de las empresas. Toda empresa constructora requiere procedimientos preestablecidos para neutralizar eventos de accidente de manera rápida y eficaz, requiriéndose para ello cálculos y análisis de riesgos que anticipen el probable suceso, de esta manera se preparan acciones específicas para contrarrestar la eventualidad (Hallowell y Gambatese, 2009). 
En la industria de la construcción, es imperativo realizar la identificación de peligros, valoración de riesgos y su priorización alcanzando soluciones viables para los trabajadores. Dada la similitud de los peligros de la construcción en los diferentes países, se puede recurrir a herramientas como la inspección e investigación para la intervención de estos (NIOSH, 2009). De ahí la inquietud de comprender las causas que producen dichos accidentes que afectan la seguridad laboral en el mundo (López et al., 2011). Los datos de seguridad laboral en los últimos diez años indican que la frecuencia de incidentes y el número de muertes en accidentes en el trabajo se han reducido en el mundo, sin embargo, es preocupante que aun persistan accidentes fatales en las construcciones por todo el mundo, siendo necesario intervenir este sector, evaluar sus riesgos e implementar soluciones en fuente y medio de propagación (Ye et al., 2016). Esta investigación cobra importancia porque su objetivo es dar tratamiento a un factor que producía daños y perjuicios a la calidad de vida de los trabajadores y a la imagen corporativa de la empresa. En el ámbito social la presente investigación promoverá la importancia de manejar las estadísticas de accidentalidad que prioricen los riesgos más relevantes tratando enfermedades laborales y accidentes de trabajo, para así prevenir la aparición de los mismos.

Proyectos de vivienda residencial experimentaron una mayor proporción de accidentes en los Estados Unidos donde más del $80 \%$ de los accidentes ocurridos por caídas fueron desde una altura inferior a 9,1 m (30 pies), y sólo el $11 \%$ de las víctimas de accidentes estaban debidamente equipados con protección contra caídas (Kang et al., 2017). Un estudio realizado en Tenessee Estados Unidos, se halló que el $66,7 \%$ de las empresas investigadas contaban con un sistema instituido de indicadores adelantados, pero el $80 \%$ de los encuestados, se relacionaron con el manejo de equipos de protección personal y programas de abuso de sustancias. Las empresas más grandes tenían más probabilidades de utilizar indicadores pasivos de seguridad relacionados con la formulación de políticas y los programas estratégicos que las empresas más pequeñas (Akroush y El-Adaway, 2017). Mientras que en España una gran empresa no siempre es necesariamente más segura que una pequeña empresa en cuanto a accidentes mortales, los trabajadores experimentados no tienen las mejores tasas de accidentes y los accidentes que ocurren lejos del lugar de trabajo habitual tienen consecuencias más graves (López et al., 2012).

\section{MATERIALES Y MÉTODOS}

Se ha desarrollado una investigación descriptiva para describir las características y los factores predominantes del fenómeno de accidentalidad en empresas de construcción. Buscando especificar las actividades importantes de personas, grupos, o cualquier otro fenómeno que sea sometido a análisis, se utilizó la consulta documental de los eventos de accidente registrados durante los años 2014, 2015 y 2016. Analizando aspectos relacionados con el accidente de trabajo discriminando variables sociodemográficas, de la muestra a examinar, además de identificar las causas que produjeron estos eventos de características negativas. Esta información fue facilitada por la empresa para el reporte de accidentes de trabajo en el intervalo de 2012 a 2013.

Por ultimo consecuente con la información recibida se planea realizar el análisis de accidentalidad que brinde recomendaciones y opciones de mejora para la prevención de accidentes y enfermedades laborales. Para la realización de esta investigación se ha considerado 118 datos de accidentalidad del personal perteneciente a empresas de construcción como fuente primaria para el progreso e impulso de la investigación, esta información se manejará en forma estadística para determinar los factores que más afectan a la salud de los trabajadores por estar expuestos a riesgos propios de las funciones de sus tareas.

\section{RESULTADOS}

Al iniciar la evaluación se organizaron por empresas y casos registrados de accidentes por año, conforme a la Tabla 1.

Tabla 1: Comportamiento de la accidentalidad por empresa

\begin{tabular}{|c|c|c|}
\hline $\begin{array}{c}\text { Empresas de } \\
\text { construcción }\end{array}$ & $\begin{array}{c}\text { Casos de accidentes } \\
\text { por año }\end{array}$ & $T=118$ \\
\hline \multirow{3}{*}{ Empresa 1 (21\%) } & 2014 & $8(7 \%)$ \\
\cline { 2 - 3 } & 2015 & $6(5 \%)$ \\
\cline { 2 - 3 } & 2016 & $11(9 \%)$ \\
\hline \multirow{3}{*}{ Empresa 2 (22\%) } & 2014 & $8(7 \%)$ \\
\cline { 2 - 3 } & 2015 & $12(10 \%)$ \\
\cline { 2 - 3 } & 2016 & $6(5 \%)$ \\
\hline
\end{tabular}


Tabla 1(continuación)

\begin{tabular}{|c|c|c|}
\hline \multirow{3}{*}{ Empresa 3 (19\%) } & 2014 & $10(8 \%)$ \\
\cline { 2 - 3 } & 2015 & $7(6 \%)$ \\
\cline { 2 - 3 } & 2016 & $5(4 \%)$ \\
\hline \multirow{3}{*}{ Empresa 4 (13\%) } & 2014 & $11(9 \%)$ \\
\cline { 2 - 3 } & 2015 & $2(2 \%)$ \\
\cline { 2 - 3 } & 2016 & $2(2 \%)$ \\
\hline \multirow{3}{*}{ Empresa 5 (12\%) } & 2014 & $4(3 \%)$ \\
\cline { 2 - 3 } & 2015 & $5(4 \%)$ \\
\cline { 2 - 3 } & 2016 & $5(4 \%)$ \\
\hline \multirow{3}{*}{ Empresa 6 (14\%) } & 2014 & $1(1 \%)$ \\
\cline { 2 - 3 } & 2015 & $7(6 \%)$ \\
\cline { 2 - 3 } & 2016 & $8(7 \%)$ \\
\hline
\end{tabular}

Tabla 2: Características sociodemográficas e incapacidades

\begin{tabular}{|c|l|c|}
\hline Edad promedio & 39,6 DS (8,9) & $T=118$ \\
\hline \multirow{3}{*}{ Estado civil } & Casado & $32(27 \%)$ \\
\cline { 2 - 3 } & Soltero & $22(19 \%)$ \\
\cline { 2 - 3 } & Unión libre & $64(54 \%)$ \\
\hline \multirow{3}{*}{ Estrato } & 1 & $63(53 \%)$ \\
\cline { 2 - 3 } & 2 & $41(35 \%)$ \\
\cline { 2 - 3 } & 3 & $14(12 \%)$ \\
\hline \multirow{2}{*}{ Escolaridad } & Primaria & $65(55 \%)$ \\
\cline { 2 - 3 } & Secundaria & $51(43 \%)$ \\
\cline { 2 - 3 } & Técnico/Tecnológico & $2(2 \%)$ \\
\hline \multirow{2}{*}{ Incapacidad } & $\mathrm{Si}$ & $33(28 \%)$ \\
\cline { 2 - 3 } & No & $85(72 \%)$ \\
\hline
\end{tabular}

La edad de los accidentados oscila los 39 años en promedio (DS 8.6), siendo los más propensos al accidente aquellos que viven en unión libre (54\%), del primer estrato socioeconómico $(53 \%)$, más prolíficos al accidente aquellos obreros con nivel primario de educación (55\%), mientras los técnicos y tecnólogos presentan menos tendencia al accidente (2\%). Respecto a la frecuencia de accidentes de trabajo (IFAT) arrojó como resultado que por cada 100000 horas hombre trabajadas ocurren 40,4 accidentes de trabajo, aunque, además se tiene a la frecuencia de accidentes incapacitantes (IFAI) que indicó que por cada 100000 horas hombres trabajadas ocurren 11.3 accidentes con incapacidad y 29.1 accidentes sin incapacidad. El año 2014 se registró la mayor accidentalidad de los periodos evaluados con un $36 \%$ del total registrado como se muestra en la Tabla 2, teniendo al cargo de ayudante como el más afectado en los años 2014 (50\%) y $2016(41 \%)$ respectivamente; 2015 obtuvo un 33\% del total de accidentes con operadores accidentados en el orden del $31 \%$ para ese año; mientras los ayudantes en el año 2016 con un $41 \%$ de los eventos registrados fue el cargo con mayor frecuencia en accidentalidad.

Tabla 3: Accidentalidad por cargos en Construcción

\begin{tabular}{|l|c|c|c|c|c|c|c|c|}
\hline \multirow{2}{*}{ Cargos } & \multicolumn{7}{|c|}{ Accidentalidad por cargo } \\
\cline { 2 - 11 } & 2014 & $\%$ & 2015 & $\%$ & 2016 & $\%$ & TOTAL & $\%$ \\
\hline Ayudante & 21 & $50 \%$ & 10 & $26 \%$ & 15 & $41 \%$ & 46 & $39 \%$ \\
\hline Operador & 8 & $19 \%$ & 12 & $31 \%$ & 9 & $24 \%$ & 29 & $25 \%$ \\
\hline Mampostero & 6 & $14 \%$ & 3 & $8 \%$ & 2 & $5 \%$ & 11 & $9 \%$ \\
\hline Carpintero & 0 & $0 \%$ & 2 & $5 \%$ & 1 & $3 \%$ & 3 & $3 \%$ \\
\hline Almacenista & 0 & $0 \%$ & 3 & $8 \%$ & 1 & $3 \%$ & 4 & $3 \%$ \\
\hline Electricista & 7 & $17 \%$ & 4 & $10 \%$ & 3 & $8 \%$ & 14 & $12 \%$ \\
\hline Pailero & 0 & $0 \%$ & 5 & $13 \%$ & 6 & $16 \%$ & 11 & $9 \%$ \\
\hline Total & 42 & $36 \%$ & 39 & $33 \%$ & 37 & $31 \%$ & 118 & $100 \%$ \\
\hline
\end{tabular}


En la Tabla 4 se ilustran los tipos de lesiones registradas, los golpes en diversas partes del cuerpo tuvieron la mayor prevalencia entre los registros manejados, con frecuencias del $50 \%$ para el $2014,45 \%$ en 2015 y $52 \%$ en 2016 respectivamente, sin embargo, estos eventos no representaron una severidad importante, pero si incapacidades temporales. Cabe resaltar que eventos como fracturas se repitieron con baja frecuencia $14 \%$ en 2014 y $10 \%$ en 2016, pero con incapacidades que pueden alcanzar hasta los tres meses.

Tabla 4: Tipo de lesiones registradas

\begin{tabular}{|c|c|c|c|c|c|c|c|c|}
\hline Tipo de lesión & 2014 & $\%$ & 2015 & $\%$ & 2016 & $\%$ & TOTAL & $\%$ \\
\hline Golpe & 21 & $50 \%$ & 19 & $45 \%$ & 22 & $52 \%$ & 62 & $53 \%$ \\
\hline Herida & 6 & $14 \%$ & 2 & $5 \%$ & 2 & $5 \%$ & 10 & $8 \%$ \\
\hline Efectos de la electricidad & 1 & $2 \%$ & 0 & $0 \%$ & 0 & $0 \%$ & 1 & $1 \%$ \\
\hline Torcedura, esguince & 2 & $5 \%$ & 3 & $7 \%$ & 1 & $2 \%$ & 6 & $5 \%$ \\
\hline Luxaciones & 2 & $5 \%$ & 7 & $17 \%$ & 0 & $0 \%$ & 9 & $8 \%$ \\
\hline Trauma superficial & 3 & $7 \%$ & 0 & $0 \%$ & 6 & $14 \%$ & 9 & $8 \%$ \\
\hline Lesiones múltiples & 1 & $2 \%$ & 1 & $2 \%$ & 1 & $2 \%$ & 3 & $3 \%$ \\
\hline Quemadura & 0 & $0 \%$ & 3 & $7 \%$ & 1 & $2 \%$ & 4 & $3 \%$ \\
\hline Cuerpo extraño & 0 & $0 \%$ & 2 & $5 \%$ & 0 & $0 \%$ & 2 & $2 \%$ \\
\hline Fractura & 6 & $14 \%$ & 2 & $5 \%$ & 4 & $10 \%$ & 12 & $10 \%$ \\
\hline Total & 42 & & 39 & & 37 & & 118 & \\
\hline
\end{tabular}

De igual manera la Tabla 4, detalla la severidad o gravedad de los accidentes de trabajo (la relación entre el número de días perdidos y cargados por los accidentes de trabajo durante el año), en la recolección de la información del periodo 2014-2016 esta fue mínima en un orden del $41 \%$, menor en un $48 \%$ y solo se registró una mayor severidad en un 11\%. En 2014 se encontró también la severidad más elevada en el nivel mayor con un $21 \%$ la relevancia existente en este indicador es por la facultad de analizar el total de días perdidos debidos a los accidentes y el total de horas-hombre de exposición al riesgo. El 2014 demostró ser el año con más días perdidos por accidentes, con lo cual se entiende que en este periodo se incurrió en más pérdidas por reemplazos de incapacitados e inhabilitados producto de los accidentes. Entre los periodos estudiados y empresas involucradas no se reportaron fatalidades. El año 2016 obtuvo el comportamiento más favorable en cuanto a la severidad a analizar pues solo reportó un $2 \%$ de severidad mayor (el indicador que enfatiza más días perdidos por prolongación de incapacidades).

Tabla 5: Accidentalidad por grado de severidad

\begin{tabular}{|l|c|c|c|c|c|c|c|c|}
\hline Severidad & 2014 & $\%$ & 2015 & $\%$ & 2016 & $\%$ & \multicolumn{3}{|c|}{ Total } \\
\hline Mínima & 18 & $43 \%$ & 17 & $40 \%$ & 13 & $31 \%$ & 48 & $41 \%$ \\
\hline Menor & 15 & $36 \%$ & 19 & $45 \%$ & 23 & $55 \%$ & 57 & $48 \%$ \\
\hline Mayor & 9 & $21 \%$ & 3 & $7 \%$ & 1 & $2 \%$ & 13 & $11 \%$ \\
\hline Mortal & 0 & $0 \%$ & 0 & $0 \%$ & 0 & $0 \%$ & 0 & $0 \%$ \\
\hline Total & 42 & & 39 & & 37 & & 118 & $100 \%$ \\
\hline
\end{tabular}

\section{DISCUSIÓN}

En esta investigación se detectaron situaciones de peligro como los golpes y traumas alrededor del 53\% coincidiendo con Gürcanli y Müngen (2013), quienes obtuvieron una prevalencia en estos eventos del 54,1\% y golpeadas por objetos lanzados o caídos a razón del 12,9\% mientras estas situaciones fueron detectadas en la presente investigación en el orden del 13\% por torceduras y heridas identificadas, lo cual indica que en el sector de la construcción se hace evidente la generación de situaciones de peligro en el aspecto mecánico, por traumas de golpeado por y golpeado contra más la caída de objetos. Estos autores consideraron el accidente fatal en operarios no calificados junto a los carpinteros, pintores, yeseros y soldadores como importante, aunque para el caso de la actual investigación no se manejaron dichas dimensiones entre sus resultados, sin embargo, las precisiones sobre caídas mortales de trabajadores permiten establecer un análisis más detallado las causas relacionadas con la construcción proporcionando información crítica para 
prevenir muertes en los sitios de construcción en cualquier lugar del mundo, por lo cual la solicitud de sistemas de acceso y elementos de protección contra caídas son medidas de prevención y protección pertinentes. Es pertinente mencionar que no solo la falta de elementos de protección personal puede desencadenar accidentes en las áreas de labores en el sector de la construcción, pues se debe mencionar que la omisión en el uso de estos elementos es precursor de más accidentes en las áreas de trabajo que la ausencia de los elementos mismos (Bedoya, 2015).

Kanchana et al. (2015) estableció en sus indagaciones que la muerte de trabajadores se genera en alrededor del $1,60 \%$ de las construcciones y situaciones como colapso de edificaciones en construcción, variedad de caídas de trabajadores desde alturas, trabajo en máquinas y hasta voladura con explosivos; contraria a la actual investigación donde no se detectaron muertes, pero si fracturas en un valor del $10 \%$, muy similar a la hallada por los autores ya mencionados con un 10,47\% inferiores a los mecanismos de accidente que aparecieron en actual investigación (golpe, luxación y fractura) menos severos pero con mayor frecuencia de aparición, con al rededor del $71 \%$. De igual forma el promedio de edad de accidentados fue 32 años registrados según ya mencionado estudio de los hindúes, sin embargo, se obtuvo un promedio de edad superior en la actual investigación, donde los promedios de los afectados tenían edades cercanas a los 40 años. Otra coincidencia con el presente estudio radica en el incumplimiento de las normas de seguridad laboral, lo que genera numerosos accidentes en los cargos de (ayudante y operadores) que tienen lugar en las obras de construcción que al carecer de directrices de la salud y la seguridad de los trabajadores que orienten medidas preventivas y el desarrollo de un ambiente de trabajo seguro, se ha abocado a las situaciones de riesgo y accidentes presentes en las estadísticas presentadas.

Se coincide en la afirmación de Chan et al. (2008) al indicar que los accidentes de trabajo en la industria de la construcción son frecuentes, provocados por el uso de andamios y escaleras para tareas de construcción en altura. También es referida la peligrosidad que revisten los andamios y escaleras pueden ser muy peligrosas y generan discapacidades permanentes, aunque no se comparten resultados en relación a una alta tasa de muertes debido a que en este estudio no hubo trabajadores fallecidos, pero si eventos ocurridos como fracturas y traumas alrededor del $18 \%$ de los eventos estudiados, provocados por las condiciones físicas de los lugares de trabajo. Pero totalmente de acuerdo con Dąbrowski (2015) quien indicó en su investigación que la industria de la construcción en Europa clasifica como la actividad con más altos riesgos laborales, en el actual estudio se ratifica esta presunción al encontrar a trabajadores con insatisfacción de seguridad laboral y la totalidad de las empresas son pequeñas y medianas las e incumpliendo las disposiciones de seguridad necesarias para que trabajos en construcción se realicen de forma segura.

No se logró coincidir con los autores Rameezdeen y Elmualim (2017) al conseguir identificar el promedio de edad de los afectados en accidentes de trabajo con un promedio de edad 39.6 años mientras estos primeros detectaron representaciones altas trabajadores que sufrieron accidentes con edades de 35 años y mayores de 55 años entre los accidentes indagados, aunque en ambas indagaciones situaciones del entorno que logran predisponer la aparición del accidente, el cual se motiva e incrementa en épocas de verano intenso, aunque en la actual investigación todos los afectados estudiados fueron varones que viven en una ciudad de altas temperaturas como Cartagena de indias.

Si se observan las condiciones locativas y meteorológicas tal como lo hicieron (Nadhim et al., 2016) quienes afirman que el $25 \%$ de los estudios señalan a los andamios y escaleras como espacios riesgosos que podría causar muertes de trabajadores de este sector aunque en el presente estudio solo se logró evidenciar la aparición de accidentes leves como torceduras, lesiones y traumas a razón del 16\%, que consolidad a las actividades de la construcción como la generadora de eventos significativos, aunque con poca severidad en el daño e incluso nula fatalidad para este estudio y de igual importancia la consideración para toda empresa constructora en el establecimiento de procedimientos de seguridad que neutralicen la ocurrencia de estos de forma eficiente y le preparan acciones específicas para contrarrestar la posible eventualidad (Hallowell y Gambatese, 2009) que además versan sobre las condiciones de las áreas de trabajo y superficie pueden afectar a los que trabajan cuando hay defectos en la superficie de trabajo. Por otro lado, los sitios de construcción a veces funcionan durante veinticuatro horas con condiciones insuficientes siendo más precaria la vida de los trabajadores que al ser afectados con lesiones como fracturas, trauma y contusiones, que pueden llevar a la muerte o en otro caso generar una lesión física (secuela) con influencia directa en el cuerpo del trabajador que en algunos casos, provocar la pérdida de una oportunidad de trabajo o de tener una discapacidad permanente.

\section{CONCLUSIONES}

De los resultados presentados, de su discusión y de los antecedentes de la literatura expuestos a través del artículo, se pueden obtener las siguientes conclusiones principales: 
Los accidentes de trabajo en el sector de la construcción son una amenaza constante para los trabajadores que se desempeñan en ella, pues ocasionan daños a la empresa y amenazas contra la vida del trabajador, causa ausentismo laboral, disminuyendo su producción y aumentando costos para la empresa por incapacidad. Los accidentes derivada o asociada a la es un problema de gran magnitud. Son los trabajadores del nivel primario de escolaridad, en unión libre y desempeñando los cargos de ayudante y operador los más accidentados de este estudio. Golpes (golpeado por y golpeado contra) tuvieron la mayor prevalencia con frecuencias del $50 \%, 45 \%$ y $52 \%$ en los respectivos años en que se desarrolló el estudio. 2014 Reportó mayor cantidad de accidentes con fractura (14\%), siendo estos los más incapacitantes del grupo de eventos de accidente analizados.

Las severidades mínimas con un $41 \%$ reflejaron que a pesar de la frecuencia de estos eventos el tiempo de incapacidad fue reducido para los periodos objeto de estudio. Se debe proponer métodos proactivos como estrategia óptima para reducir accidentes fundamentados en educar y capacitar a los trabajadores de la construcción también puede reducir eventos graves. Además, el diseño de cursos breves de capacitación en seguridad para los trabajadores, seminarios y charlas enfocadas en el trabajo en construcción, para tener impactos positivos en el comportamiento de los trabajadores de la industria de la construcción que deben están sujetas a normas / directrices específicas de seguridad, sometidos a su vez a regulaciones nacionales e internacionales de seguridad y salud en el trabajo para mejorar la conducta de operarios in situ y prevenir las lesiones mientras se trabaja.

\section{REFERENCIAS}

Akroush, N.S., y El-Adaway, I.H. Utilizing construction leading safety indicators: Case study of tennessee. Doi:10.1061/(ASCE)ME.1943-5479.0000546. Journal of Management in Engineering (en línea), 33(5) (2017)

Alizadeh, S.S., Mortazavi, S.B., y Sepehri, M.M. Analysis of Occupational Accident Fatalities and Injuries Among Male Group in Iran Between 2008 and 2012. Iranian Red Crescent Medical Journal, 17(10), (2015)

Bedoya, E., Severiche, C., Sierra, D. y Sierra, C. Integrated Management System Based on the OHSAS 18001: 2007 and ISO 28000: 2006 Standards for a Logistic Services Company. International Journal of Engineering and Applied Sciences, 4(7), 85- 89 (2017)

Bedoya, EA., Manual de trabajo en altura, 10-12. Grupo Editorial Alfa Omega, Cartagena, Colombia (2015)

Chan, A.P., Wong, F.K., Chan, D.W., Yam, M.C., Kwok, A.W., Lam, E.W., Cheung, E. Work at height fatalities in the repair, maintenance, alteration, and addition works. J. Constr. Eng. Manag., 134, 527-535 (2008)

Dąbrowski, A. An investigation and analysis of safety issues in Polish small construction plants. International Journal of Occupational Safety and Ergonomics, 21(4), 498-511 (2015)

Fang, D.P, Huang, X.Y., Hinze J. Benchmarking studies on construction safety management in China. J. Cons. Eng. Man., 130, 424-32 (2004)

Gómez, P., Gómez, E., Severiche, C. Riesgos físicos en vendedores minoristas del Mercado de Bazurto, Caribe Colombiano. Ciencia y Salud Virtual, 9(1), 13-22 (2017)

Gürcanli, G. y Müngen, U. Analysis of Construction Accidents in Turkey and Responsible Parties. Industrial Health, 51(6), 581-595 (2013)

Hallowell, M.R., Gambatese J.A. Construction Safety Risk Mitigation. J. Constr. Eng. Manag. 135, 13161323 (2009)

Kanchana, S., Sivaprakash, P. y Joseph, S. Studies on Labour Safety in Construction Sites. The Scientific World Journal (2015)

Kang, Y., Siddiqui, S., Suk, S.J., Chi, S. y Kim, C. Trends of fall accidents in the U.S. construction industry doi:10.1061/(ASCE)CO.1943-7862.0001332. Journal of Construction Engineering and Management (en línea), 143(8) (2017)

López Arquillos, A., Rubio Romero, J.C. y Gibb, A. Analysis of construction accidents in spain, 2003-2008. doi:10.1016/j.jsr.2012.07.005. Journal of Safety Research (en línea), 43(5-6), 381-388 (2012) 
Mehrdad, R., Seifmanesh, S., Chavoshi, F., Aminian, O., y Izadi, N. Epidemiology of Occupational Accidents in Iran Based on Social Security Organization Database. Iranian Red Crescent Medical Journal, 16(1), e10359 (2014)

Nadhim, E.A., Hon, C., Xia, B., Stewart, I., y Fang, D. Falls from Height in the Construction Industry: A Critical Review of the Scientific Literature. International Journal of Environmental Research and Public Health, 13(7), 638 (2016)

NIOSH. Qualitative risk characterization and management of occupational hazards: Control Banding (CB); a literature review and critical analysis. Cincinnati $(\mathrm{OH})$ : National Institute for Occupational Safety and Health (US); Report No: 2009-152 (2009)

Rameezdeen, R., y Elmualim, A. The Impact of Heat Waves on Occurrence and Severity of Construction Accidents. International Journal of Environmental Research and Public Health, 14(1), 70 (2017)

Work-Related Injuries and Fatalities Involving a Fall from Height, Australia. En línea: https://goo.gl/4AscK6. ISBN [PDF] 978-1-74361-187-6, [DOCX] 978-1-74361-188-3. Acceso: 31 de Octubre (2013)

Ye, Y., Zhang, S., Rao, J., Wang, H., Li, Y., Wang, S., y Dong, X. Analysis of National Major Work Safety Accidents in China, 2003-2012. Iranian Journal of Public Health, 45(1), 6-13 (2016)

Vega, N., Bedoya, E., Sierra, D. y Severiche, C. Niveles de presión sonora en una empresa de maquinaria pesada: estrategias administrativas y técnicas para su disminución. Revista Ingenierías USB Med. 8(2), 23 $30(2017)$ 